\title{
A Functionally Conserved Mechanism of Modulation via a Vestibule Site in
}

\section{Pentameric Ligand-Gated Ion Channels}

4 Marijke Brams ${ }^{1}$, Cedric Govaerts ${ }^{2}$, Kumiko Kambara ${ }^{3}$, Kerry Price ${ }^{4}$, Radovan

5 Spurny $^{1}$, Anant Gharpure ${ }^{5}$, Els Pardon ${ }^{6,7}$, Genevieve L. Evans ${ }^{1}$, Daniel Bertrand ${ }^{3}$,

6 Sarah C. R. Lummis ${ }^{4}$, Ryan E. Hibbs ${ }^{5}$, Jan Steyaert ${ }^{6,7}$ and Chris Ulens ${ }^{1}$

$8{ }^{1}$ Laboratory of Structural Neurobiology, Department of Cellular and Molecular

9 Medicine, Faculty of Medicine, KU Leuven, 3000 Leuven, Belgium

102 Laboratory for the Structure and Function of Biological Membranes, Center for

11 Structural Biology and Bioinformatics, Université libre de Bruxelles, 1050 Brussels,

12 Belgium

$13{ }^{3}$ HiQscreen, 1222 Vésenaz, Geneva, Switzerland

$14{ }^{4}$ Department of Biochemistry, University of Cambridge, Cambridge CB2 1QW,

15 United Kingdom

$16{ }^{5}$ Departments of Neuroscience and Biophysics, University of Texas Southwestern

17 Medical Center, Dallas, Texas 75390, USA

$18{ }^{6}$ Structural Biology Brussels, Vrije Universiteit Brussel, 1050 Brussels, Belgium

${ }^{7}$ VIB-VUB Center for Structural Biology, VIB, 1050 Brussels, Belgium 


\section{ABSTRACT}

Pentameric ligand-gated ion channels (pLGICs) belong to a class of ion 29 channels involved in fast synaptic signaling in the central and peripheral nervous 30 systems. Molecules acting as allosteric modulators target binding sites that are remote 31 from the neurotransmitter binding site, but functionally affect coupling of ligand binding 32 to channel opening. Here, we investigated an allosteric binding site in the ion channel 33 vestibule, which has converged from a series of studies on prokaryote and eukaryote 34 channel homologs. We discovered single domain antibodies, called nanobodies, 35 which are functionally active as allosteric modulators, and solved co-crystal structures 36 of the prokaryote channel ELIC bound either to a positive (PAM) or a negative (NAM)

37 allosteric modulator. We extrapolate the functional importance of the vestibule binding 38 site to eukaryote ion channels, suggesting a conserved mechanism of allosteric 39 modulation. This work identifies key elements of allosteric binding sites and extends 40 drug design possibilities in pLGICs using nanobodies. 


\section{INTRODUCTION}

In 1965, Monod, Wyman and Changeux postulated the model of allosteric modulation in proteins (Monod, Wyman, and Changeux 1965). According to this model, proteins exist in two possible conformational states, the tensed $(T)$ or relaxed state (R). The substrate typically has a high affinity for the $T$ state. In multi-subunit proteins, all subunits undergo a concerted transition from the $\mathrm{R}$ to the $\mathrm{T}$ state upon substrate binding. The equilibrium can be shifted to the $\mathrm{R}$ or the $\mathrm{T}$ state through $\mathrm{a}$ ligand that binds at a site that is different from the substrate binding site, in other words an allosteric site.

Changeux subsequently devoted much of his scientific career to the study of allosteric proteins, with specific attention begin paid to the nicotinic acetylcholine receptor (nAChR). This protein is a ligand-gated ion channel (LGIC) and thus in effect has no substrate, but the principle of allosteric modulation is similar in that binding of acetylcholine (ACh) shifts the thermodynamic equilibrium from a closed channel state to an open channel state through binding to a site $\sim 50 \AA$ from the channel gate. The $\mathrm{nAChR}$ is a member of a superfamily of pentameric LGICs (pLGICs), which play major roles in fast synaptic transmission in the central and peripheral nervous systems, and are the site of action of many therapeutic drugs.

Structures of these proteins have been elucidated over the last decade and several $\mathrm{nAChR}$ structures are now available for the heteromeric $\alpha 4 \beta 2 \mathrm{nAChR}$ (Morales-Perez, Noviello, and Hibbs 2016; Walsh et al. 2018), as well as other members of the pLGIC family, including the 5- $\mathrm{HT}_{3}$ serotonin receptor (Hassaine et al. 2014; Polovinkin et al. 2018; Basak et al. 2018), the glycine receptor (Du et al. 2015; Huang et al. 2015), the $\mathrm{GABA}_{\mathrm{A}}$ receptor (Zhu et al. 2018; Phulera et al. 2018; Laverty et al. 2019; Masiulis et al. 2019; Miller and Aricescu 2014), the glutamate-gated chloride channel from $C$. elegans GluCl (Hibbs and Gouaux 2011; Althoff et al. 2014) and the prokaryote channels ELIC (Hilf and Dutzler 2008) and GLIC (Hilf and Dutzler 2009; Bocquet et al. 2009). Historically, crucial structural insight into the class of nicotinic receptors was derived from cryo-electron microscopic structures of the nAChRs from the electric organ of Torpedo (Miyazawa, Fujiyoshi, and Unwin 2003; Unwin 2005) and X-ray crystal structures of the acetylcholine binding protein (AChBP; found in certain snails and worms), which is homologous to the extracellular ligand binding domain (LBD) of nAChRs (Brejc et al. 2001). 
The concept of allosteric modulation is now also more broadly applied to understand the mode of action of certain drugs, called allosteric modulators, which bind at a site that is different from the neurotransmitter binding site, but which can alter energy barriers between multiple conformational states (Bertrand and Gopalakrishnan 2007). For example, in the case of pLGICs, positive allosteric modulators (PAMs) of the nAChR can facilitate a transition from a resting to an activated state, thus enhancing the agonist-evoked response. In contrast, negative allosteric modulators (NAMs) hinder such a transition, thus diminishing the agonist response. From a drug development perspective, PAMs or NAMs are highly attractive as they finely tune receptor activation without affecting the normal fluctuations of neurotransmitter release at the synapse. One of the most extensively described PAMs used in clinic are the benzodiazepines, which act on $\mathrm{GABA}_{\mathrm{A}}$ receptors and are widely prescribed as hypnotics, anxiolytics, anti-epileptics or muscle relaxants. Important insights into the molecular recognition of these modulators are now revealed by high resolution structural data (Zhu et al. 2018; Phulera et al. 2018; Laverty et al. 2019; Masiulis et al. 2019; Miller and Aricescu 2014).

With the availability of a growing amount of structural data for these receptors, a diverse array of molecules has been revealed, many of which bind at distinct allosteric binding sites, including general anesthetics (Nury et al. 2011; Sauguet et al. 2013; Spurny et al. 2013), neurosteroids (Miller et al. 2017; Laverty et al. 2017; Q. Chen et al. 2018), lipids (Zhu et al. 2018; Laverty et al. 2019), antiparasitics (Hibbs and Gouaux 2011; Althoff et al. 2014), and many others (Nys et al. 2016). Detailed investigation of allosteric sites not only brings further knowledge about the receptor functionality but also uncovers novel drug target sites. However, our current understanding of this multi-site mechanism of allosteric modulation in pLGICs is still incomplete.

In this study, we used complementary structural and functional approaches to expand our understanding of the molecular mechanism of allosteric modulation in pLGICs. Using the prokaryote ELIC channel as a model, we explored the potential of nanobodies (single chain antibodies) as allosteric modulators. We discovered functionally active nanobodies, which act either as a PAM or NAM on ELIC and determined co-crystal structures to elucidate the nanobody interactions with ELIC. One of the structures reveals an allosteric binding site located near the vestibule of the extracellular ligand-binding domain. Comparison of conservation and divergence 
in this site in different prokaryotic and eukaryotic receptors suggests a mechanism for achieving subtype-selective allosteric modulation across the receptor superfamily. Using cysteine-scanning mutagenesis and electrophysiological recordings we extrapolate to how the vestibule site can also be targeted for modulation of the human $5-\mathrm{HT}_{3 \mathrm{~A}}$ receptor as a proof of principle relevant to other eukaryotic receptors.

\section{RESULTS AND DISCUSSION}

\section{Identification of nanobodies active as allosteric modulators on ELIC}

In this study, we took advantage of nanobodies, which are high affinity single chain antibodies derived from llamas; they have been widely employed to facilitate structural studies (Manglik, Kobilka, and Steyaert 2017) and also hold potential as therapeutics against many possible targets. A first example, caplacizumab (Cabilivi ${ }^{\circledR}$ ), has recently reached the market (Scully et al. 2019). Using the prokaryote ion channel ELIC as a model system, we investigated whether nanobodies could be selected with allosteric modulator activity on ligand-gated ion channels. We expressed ELIC channels in Xenopus oocytes and employed automated electrophysiological recordings to characterize a panel of more than 20 different ELIC nanobodies. While none of the nanobodies had any functional effect on ELIC when applied alone, we found that co-application with the agonist GABA evoked a response that broadly falls into 3 categories. One type of nanobodies enhanced the agonist-evoked response, while a $2^{\text {nd }}$ type of nanobodies diminished the agonist-evoked response and the $3^{\text {rd }}$ type had little to no effect. From these, we selected several enhancers (PAMs) and inhibitors (NAMs) for a detailed electrophysiological characterization as potential allosteric modulators. In parallel, we conducted X-ray diffraction screening of ELIC plus nanobody co-crystals for structural elucidation. From this selection, we obtained a PAM-active nanobody (PAM-Nb) as well as another NAM-active nanobody (NAM$\mathrm{Nb}$ ) and determined their structures bound to ELIC by X-ray crystallography.

Co-application of the agonist GABA with a range of PAM-Nb concentrations demonstrates that PAM-Nb enhances the agonist response (Fig. 1a) with a pEC $\mathrm{C}_{50}$ value of $5.37 \pm 0.03\left(E C_{50}: 4.2 \mu \mathrm{M}\right)$ and $I_{\max }=257 \pm 14 \%(n=8)$. In contrast, co-application of a range of NAM-Nb concentrations demonstrates that NAM-Nb decreases the agonist response (Fig. 1b) with a $\mathrm{plC}_{50}$-value of $6.89 \pm 0.03\left(\mathrm{IC}_{50}: 0.13 \mu \mathrm{M}\right)$ and 
$I_{\max }=34 \pm 2 \% \%(n=6)$. Unlike competitive antagonists, which fully inhibit the agonist response at saturating concentrations, the inhibition of NAM-Nb levels off at $70 \%$ of the response with GABA alone, consistent with the mode of action of certain NAMs. These results demonstrate that functionally active nanobodies can be developed against the ligand-gated ion channel ELIC.

\section{Crystal structures of ELIC in complex with a PAM- or NAM-type nanobody}

To gain insight into the structural recognition of positive (PAM-Nb) or negative (NAM-Nb) allosteric modulators we solved X-ray crystal structures of ELIC in complex with the PAM-Nb or NAM-Nb, respectively. The structure of ELIC in complex with the PAM-Nb was determined at a resolution of $2.59 \AA$, and reveals five PAM-Nb molecules bound to a single pentameric ELIC channel (Fig. 1c,e). Each PAM-Nb binds at an intrasubunit site in the ELIC extracellular ligand binding domain. When viewed from the top along the 5-fold symmetry axis the nanobodies extend outward and the structure resembles a 5-bladed propeller (Fig. 1c). The structure of ELIC in complex with NAM-Nb was determined at a resolution of $3.25 \AA$ and is structurally distinct from the complex with PAM-Nb: instead of five $\mathrm{Nb}$ molecules bound to the ELIC pentamer, here a single NAM-Nb molecule is bound to the ELIC pentamer (Fig. 1d,f). The NAM$\mathrm{Nb}$ binds at the channel vestibule entrance and near to the $\mathrm{N}$-terminal $\alpha$-helix of a single ELIC subunit, and is oriented in such a manner that only a single nanobody molecule can bind at this interface, as the core of the nanobody sterically hinders access to the 4 other sites (Fig. 1d,f).

A more detailed analysis of the interaction interface between both nanobodies and ELIC reveals remarkable features (Fig. 2). The PAM-Nb binds to the extracellular ligand-binding domain and forms extensive interactions through the complementarity determining region CDR1 (residues S25-I33) of the nanobody (Fig. 2a). The tip of the finger of this loop region wedges in between the ELIC $\beta 8$ - and $\beta 9$-strand, forming a distinct anti-parallel $\beta$-sheet interaction with the $\beta 8$-strand. The CDR1 loop region

171 points toward an allosteric binding site previously identified in a chimera of the human

$172 \alpha 7 \mathrm{nAChR}$ and the acetylcholine binding protein, $\alpha 7-\mathrm{AChBP}$ (Li et al. 2011) (see 173 complex with fragment molecule CU2017, pdb code 5oui) (Fig. 2a). In other words, 
175 allosteric site in the human $\alpha 7 \mathrm{nAChR}$, consistent with its function as an allosteric 176 modulator.

177 The mode of interaction of the NAM-Nb with ELIC is distinct from the PAM-Nb 178 (Fig. 2b). The interaction interface is remarkable in that none of the CDR1-, CDR2- or 179 CDR3-regions interact with ELIC. Instead, it is the region P104-L110 of the NAM-Nb 180 that forms an $\alpha$-helix, and which corresponds to a region that has no defined

181

182 secondary structure in most other nanobody structures. This $\alpha$-helical region interacts with the $\alpha$ '1-helix in ELIC (N60-N69) and forms the outer rim of an allosteric binding site previously identified as the vestibule binding site (Spurny et al. 2012). This site is the target for the benzodiazepine flurazepam, which acts as a positive allosteric modulator on ELIC (Spurny et al. 2012) (Fig. 2b). Similar to the PAM-Nb, it is interesting to observe that the allosteric binding site of the NAM-Nb also overlaps with a previously identified binding site for a small molecule allosteric modulator. Remarkably, in each case, the nanobody has the opposite functional effect of the small molecule at the same site. The PAM-Nb acts as a positive modulator, whereas the CU2017 fragment acts as a negative modulator at the $\beta 8-\beta 9$ site. Conversely, the $\mathrm{NAM}-\mathrm{Nb}$ acts as a negative modulator, whereas flurazepam acts as a positive modulator at the vestibule site. This result demonstrates that the same allosteric site can be targeted both by a PAM or NAM, and that its functional action likely depends on defined side chain interactions. This is consistent with previous pharmacological studies, which have shown that a substitution as small as a methylation of an aromatic ring in a small molecule modulator can alter the functional profile from a PAM to a NAM of the $\alpha 7$ nAChR (Gill-Thind et al. 2015).

The pores of both $\mathrm{Nb}$ complexes resemble previous structures of ELIC, with narrow constriction points at the 9', 16', and 20' positions (Hilf and Dutzler 2008), suggesting that the channels are in non-conducting conformations (Fig. 2c,d). This closed state is structurally distinct from the putative desensitized state of the $\alpha 4 \beta 2$ $\mathrm{nAChR}$, whose primary gate lies at the cytoplasmic end at the -1 ' position ${ }^{3}$. Further analysis of the central channel axis of the NAM-Nb complex reveals a complete occlusion of the extracellular vestibule by the nanobody (Fig. 2d). Despite this apparent block of the ion permeation pathway, NAM-Nb only inhibits GABA-induced currents by $\sim 35 \%$, as mentioned previously. This can potentially be explained by alternate pathways for ion entry through lateral fenestrations located at subunit 
interfaces, as seen in other members of the pLGIC family (Zhu et al. 2018; Miller and Aricescu 2014).

\section{Subtype-dependent vestibule site access in different prokaryote and eukaryote} receptors

To further investigate the possible conservation of the vestibule binding site in different prokaryote and eukaryote pLGICs, we performed a systematic analysis of the vestibule site architecture in all currently available pLGIC structures. The results from this analysis show that the outer rim of the vestibule site, which corresponds to residues N60-F95 in ELIC, can adopt one of 3 possible conformations (Fig. 3).

In certain structures, we observe that the stretch of amino acids between the $\beta 4$ - and $\beta 5$-strands resembles the shape of the Greek letter $\Omega$, for example in the $\alpha 4$ nAChR subunit (Morales-Perez, Noviello, and Hibbs 2016; Walsh et al. 2018), and therefore this region is termed the $\Omega$ loop (Hu et al. 2018). In Fig. 3a, the outer rim of the vestibule site in the $\alpha 4 \mathrm{nAChR}$ is shown in blue (from the helix to the $\beta 5$-strand) and the $\Omega$ loop in red. The tip of the $\Omega$ loop can either point into the vestibule as in the a4 nAChR subunit (Morales-Perez, Noviello, and Hibbs 2016; Walsh et al. 2018), which we call the $\Omega$-in conformation (Fig. 3a), or the tip of the $\Omega$ loop can point outward, which we call the $\Omega$-out conformation, for example in the $\alpha 1$ GlyR subunit (Du et al. 2015) (outer rim shown in blue, $\Omega$-out in green, Fig. 3b). Importantly, in both of these conformations access to the vestibule site is occluded. In the $\Omega$-in conformation, the tip of the $\Omega$ loop prevents access to the vestibule site of its own $\alpha 4$ nAChR-subunit (Fig. 3a,d), whereas in the $\Omega$-out conformation vestibule access is prevented by the tip of the $\Omega$-out loop of its neighboring $\alpha 1$ GlyR (-) subunit (Fig. 3b,e). In addition to the $\Omega$-in and $\Omega$-out conformations, we observe a third possible conformation in which the $\Omega$ loop is stretched, for example in the 5-HT $3 \mathrm{~A} R$ (Hassaine et al. 2014) (Fig. 3c) and ELIC, and creates an accessible vestibule site (Fig. 3f). Consequently, we term this conformation the $\Omega$-open conformation (outer rim and $\Omega$-open is shown in blue, Fig. 3c,f).

A systematic comparison of the $\Omega$ loop conformation demonstrates that the different pLGIC subfamilies group into defined categories. First, we observe that all currently known anionic receptor structures adopt the $\Omega$-out conformation (Fig. 3h). 
240 This implies that the vestibule access is occluded in all of these receptors, for example

241 in the homomeric $\alpha 1$ GlyR (Du et al. 2015), $\alpha 3$ GlyR (Huang et al. 2015), or GluCl

242 (Hibbs and Gouaux 2011) as well as the heteropentameric $\alpha 1 \beta \gamma 2$ GABA $_{A}$ Rs (Zhu et

243 al. 2018; Phulera et al. 2018; Laverty et al. 2019) and homopentameric $\beta 3$ GABA $R$

244 (Miller and Aricescu 2014). The $\Omega$-out loop sequence is strongly conserved in these

245 receptors, with a His residue and Asn residue at either end of the $\Omega$ loop and a Thr at

246 the tip (not conserved in $\mathrm{GluCl}$ ). We also observe that in all these cases the $\Omega$ loop is

247 preceded by a stretch of amino acids that forms an additional $\beta$-sheet, which we call

248 the $\beta 4$ '-sheet, as it also follows the $\beta 4$-sheet. The $\beta 4$ '-sheet contains a well conserved

249 start Lys residue, which is present in GlyRs, GABA $A_{A}$ s and $\mathrm{GluCl}$. The sequence

250 conservation in these 2 regions suggests an important functional role. In contrast with

251 anionic receptors, we observe that certain cationic receptors can adopt either the $\Omega$ -

252 in or $\Omega$-open conformation. However, none of the cationic receptors adopt the $\Omega$-out

253 conformation. Only $3 \mathrm{nAChRs}$ adopt the $\Omega$-in conformation, namely the $\alpha 2 \mathrm{nAChR}$

254 (Kouvatsos et al. 2016), $\alpha 4$ and $\beta 2$ nAChR-subunits (Morales-Perez, Noviello, and 255 Hibbs 2016; Walsh et al. 2018). This implies that the heteropentameric $\alpha 4 \beta 2$ nAChRs

256 (Morales-Perez, Noviello, and Hibbs 2016; Walsh et al. 2018) adopt an all-subunit-

257 occluded vestibule site conformation. The stretch of amino acids that form the $\Omega$-in

258 conformation is also well conserved with an Asp-Gly and Val-Thr/Ser on either end

259 and an aromatic residue (Phe/Tyr) at the tip, again suggesting an important functional

260 role. In contrast, all other nAChR subunits (Dellisanti et al. 2007; Zouridakis et al.

261 2014), including the Torpedo nAChR (Miyazawa, Fujiyoshi, and Unwin 2003), as well

262 as the 5-HT 3 AR (Hassaine et al. 2014), snail AChBPs (Brejc et al. 2001; Celie et al.

263 2005), prokaryote GLIC (Bocquet et al. 2009; Hilf and Dutzler 2009) and ELIC adopt

264 the $\Omega$-open conformation. The sequence of amino acids forming the $\Omega$-open loop is

265 less conserved, except for the position +2 following the $\beta 4$-sheet, which is a Asn in all

266 receptors except $5-\mathrm{HT}_{3 \mathrm{~A}} \mathrm{R}$. Important to note is that the $\Omega$-open loop in the $\alpha 9 \mathrm{nAChR}$

267 (Zouridakis et al. 2014) is disordered in the apo state (pdb code 4d01), suggesting

268 flexibility in this region, but not in the antagonist (MLA)-bound state (pdb code 4uxu).

269 This raises the intriguing possibility that the $\Omega$ loop is intrinsically flexible and could

270 undergo drug-induced conformational changes, similar to loop $C$ at the

271 neurotransmitter binding site (Brams et al. 2011). 
Together, the results from this analysis demonstrate that different receptor subtypes adopt a different $\Omega$ loop conformation, with the $\Omega$-open conformation creating an accessible vestibule site, whereas the $\Omega$-in and $\Omega$-out conformations occlude vestibule site access. This discovery provides new opportunities for drug design of allosteric molecules that have subtype-specific pharmacology (due to low sequence conservation compared to the orthosteric site), based on vestibule site access.

Cysteine-scanning mutagenesis in the vestibule site of the human $5-\mathrm{HT}_{3 \mathrm{~A}}$ receptor

Based on our observations that the vestibule site in ELIC is the target for positive allosteric modulators such as the benzodiazepine flurazepam (Spurny et al. 2012) as well as negative allosteric modulators such as the NAM-nanobody described in this study, we further investigated whether the mechanism of vestibule site modulation is functionally conserved in eukaryote receptors. We chose the $5-\mathrm{HT}_{3 \mathrm{~A}} \mathrm{R}$ as a prototype receptor since it has a clear $\Omega$-open vestibule conformation and its structure (Hassaine et al. 2014) as well as functional and pharmacological properties (Lummis 2012) are well described. We then employed the substituted cysteine accessibility method (SCAM) (Karlin and Akabas 1998) to investigate the functional effects on channel gating before and after modification of cysteines in the $5-\mathrm{HT}_{3 \mathrm{~A}} \mathrm{R}$ vestibule site with the cysteine-reactive reagent MTSEA-biotin. We chose residues on the outer rim of the vestibule site, T112 (top) and F125 (bottom), respectively, as well as residues deeper into the vestibule site, N147, K149 and L151 on the $\beta 6$-strand and Y86 on the $\beta 2$-strand (Fig. 4b). It is interesting to note that N147, K149 and L151 are located on the opposite side of the $\beta$-strand to the loop $E$ residues ( $Q 146$ and $Y 148$ ), while $\mathrm{Y} 86$ is on the opposite side of the $\beta$-strand to the loop D residues (W85 and R87); both of these regions are functionally important contributors to the neurotransmitter binding site (Hassaine et al. 2014). Residue P111, which points away from the vestibule site, was included as a negative control.

Representative current traces of channel responses to increasing concentrations of serotonin (5-HT) are shown in Fig. 4a for the L151C mutant before and after modification with MTSEA-biotin. The channel responses are drastically increased after cysteine modification, which is caused by a more than 10-fold increase 
305

306

307

308

309

310

311

312

313

314

315

316

317

318

319

320

321

322

323

324

325

326

327

328

329

330

331

332

333

334

335

336

337

MTSEA-biotin modification as well as a shift of the $\mathrm{EC}_{50}$-value to lower concentrations (Fig. 4a,c) $\left(\mathrm{pEC}_{50}: 4.01 \pm 0.03, \mathrm{n}=5\left(\mathrm{EC}_{50}: 98 \mu \mathrm{M}\right)\right.$ versus $\mathrm{pEC}_{50}: 4.96 \pm 0.04, \mathrm{n}=7$ $\left.\left(\mathrm{EC}_{50}: 11 \mu \mathrm{M}\right)\right)$, indicating a strong PAM-effect at this position $(P<0.001)$. Mutants T112C and K149C also showed a significant decrease of the $\mathrm{EC}_{50}$-value after MTSEAbiotin, namely $6.7 \mu \mathrm{M}$ (pEC $50: 5.17 \pm 0.02, \mathrm{n}=4$ ) versus $2.9 \mu \mathrm{M}\left(\mathrm{pEC}_{50}: 5.53 \pm 0.02\right.$, $\mathrm{n}=4)$ for T112C (Fig. 3d) $(P<0.0001)$ and $6.2 \mu \mathrm{M}\left(\mathrm{pEC}_{50}: 5.21 \pm 0.05, \mathrm{n}=7\right)$ versus $2.1 \mu \mathrm{M}$ (pEC $50: 5.69 \pm 0.11, \mathrm{n}=7)$ for K149C (Fig. 3e) $(P<0.002)$. Although neither of these mutants displayed an increase of $I_{\max }$ as in the L151C mutant, the leftward shift of the concentration-activation curve is also consistent with a PAM-effect at these positions. No significant differences were observed for current responses before and after MTSEA-biotin for Y86C, F125C and N147C or the negative control, P111C, suggesting modification of these residues does not place the MTSEA-biotin in an appropriate position to act as a modulator, although it is also possible it did not react here. Together, these results demonstrate that allosteric modulation via the vestibule binding site is functionally conserved between ELIC and 5- $\mathrm{HT}_{3 \mathrm{~A}}$ receptors. The evolutionary distance between these receptors, combined with previous work, suggests that this mechanism of modulation likely also extends to other pLGIC members, and indeed the functional importance of the vestibule site in channel modulation is now emerging from a wide range of different studies demonstrating ELIC modulation by flurazepam (Spurny et al. 2012) or nanobodies (this study), 4bromocinnamate modulation of the prokaryote homolog STeLIC (Hu et al. 2018), as well as eukaryote $5-\mathrm{HT}_{3 \mathrm{~A}} \mathrm{R}$ modulation by cysteine-scanning mutagenesis (this study), and $\alpha 7$ nAChR modulation using a fragment-based screening approach (Spurny et al. 2015; Delbart et al. 2018).

In conclusion, we demonstrate here that functionally active nanobodies can be developed for the ligand-gated ion channel ELIC. Functional characterization demonstrates that nanobodies can act as positive or negative allosteric modulators. Crystal structures reveal that ELIC nanobodies can interact via distinct epitopes, including accessible parts of allosteric binding sites previously discovered in the extracellular ligand binding domain and bound by small molecules. One potentially attractive site for further development of allosteric modulators is the vestibule site, which can be targeted not only with nanobodies or small molecules in ELIC, but also 
by chemical modification of engineered cysteines as demonstrated in the human 5$\mathrm{HT}_{3 \mathrm{~A}}$ receptor. The vestibule site offers opportunities to further develop both positive and negative modulators, as well as to exploit subtype-specific access to certain receptors. These results pave the way for the future development of novel therapeutics that can modulate channel activity in pLGIC-related disorders. An attractive path is to expand the currently available repertoire of therapeutics with pharmacologically active nanobodies against human pLGICs.

\section{Production of nanobodies against ELIC}

A llama was immunized with $2 \mathrm{mg}$ in total of purified wild type ELIC protein over a period of 6 weeks using a previously established protocol (Pardon et al. 2014). Briefly, from the anti-coagulated blood of the immunized llama, lymphocytes were used to prepare cDNA. This cDNA served as a template to amplify the open reading frames coding for the variable domains of the heavy-chain only antibodies, also called nanobodies. The PCR fragments were ligated into the pMESy4 phage display vector and transformed in E. coli TG1 cells. A nanobody library of $1.1 \times 10^{8}$ transformants was obtained. For the discovery of ELIC specific nanobodies, wild type ELIC was solid phase coated directly on plates and phages were recovered by limited trypsinization. After two rounds of selection, periplasmic extracts were made and subjected to ELISA screens, seven different families were confirmed by sequence analysis. All clones were produced and purified as previously described (Pardon et al. 2014).

\section{Nanobody expression and purification}

A series of nanobodies were individually expressed in the periplasm of $E$. coli strain WK6, which was grown in TB media supplemented with $0.1 \mathrm{mg} / \mathrm{ml}$ carbenicillin, $0.1 \%$ glucose and $2 \mathrm{mM} \mathrm{MgCl}_{2}$ to an absorbance $\mathrm{A}_{600} \sim 0.7$ at $37^{\circ} \mathrm{C}$. The culture was induced with $1 \mathrm{mM}$ isopropyl $\beta$-D-1-thiogalactopyranoside (IPTG) and incubated in an orbital shaker overnight at $28^{\circ} \mathrm{C}$. Cells were harvested by centrifugation, resuspended in TES buffer (200 mM TRIS, pH 8.0; 0.5 mM EDTA; 500 mM sucrose supplemented with $40 \mathrm{mM}$ imidazole) and incubated for $1 \mathrm{~h}$. To this fraction 4 times diluted TES buffer was added and incubated for $1 \mathrm{~h}$. This fraction was cleared by centrifugation at 10,000 
372 g. The supernatant was incubated with Ni Sepharose 6 Fast Flow resin (GE

373 Healthcare) and incubated for $1 \mathrm{~h}$ at room temperature. The beads were washed with

374 buffer containing $20 \mathrm{mM}$ TRIS pH 8.0, $300 \mathrm{mM} \mathrm{NaCl}$ and $40 \mathrm{mM}$ imidazol. Protein was

375 eluted with the same buffer supplemented with $300 \mathrm{mM}$ imidazole. The eluted protein

376 was concentrated to less than $1 \mathrm{ml}$ on a $3 \mathrm{kDa}$ cut-off Vivaspin concentrating column

377 (Sartorius) and further purified on a Superdex 75 10/300 GL column (GE Healthcare)

378 equilibrated with $10 \mathrm{mM}$ Na-phosphate $(\mathrm{pH} \mathrm{8.0)}$ and $150 \mathrm{mM} \mathrm{NaCl}$. Peak fractions

379 corresponding to nanobody were pooled and spin-concentrated to $\sim 50 \mathrm{mg} / \mathrm{ml}$.

380

381

\section{Automated voltage-clamp recordings of ELIC}

For expression of ELIC in Xenopus oocytes we used the pGEM-HE expression plasmid (Liman, Tytgat, and Hess 1992) containing the signal sequence of the human $\alpha 7 \mathrm{nAChR}$ followed by the mature ELIC sequence, as previously described (Spurny et al. 2012). After plasmid linearization with Nhel, capped mRNA was transcribed in vitro using the mMESSAGE mMACHINE T7 transcription kit (ThermoFisher). $2 \mathrm{ng}$ of mRNA per oocyte was injected into the cytosol of stage $\mathrm{V}$ and $\mathrm{VI}$ oocytes using the Roboinject automated injection system (Multi Channel Systems). Oocyte preparations and injections were done using standard procedures (Knoflach, Hernandez, and Bertrand 2018). Injected oocytes were incubated in ND96-solution containing $96 \mathrm{mM}$ $\mathrm{NaCl}, 2 \mathrm{mM} \mathrm{KCl}, 1.8 \mathrm{mM} \mathrm{CaCl}_{2}, 2 \mathrm{mM} \mathrm{MgCl} 2$ and $5 \mathrm{mM} \mathrm{HEPES}, \mathrm{pH}$ 7.4, supplemented with $50 \mathrm{mg} / \mathrm{L}$ gentamicin sulfate. One to five days after injection, electrophysiological recordings were performed at room temperature by automated two-electrode voltage clamp with the HiClamp apparatus (Multi Channel Systems). Cells were superfused with standard OR2 solution containing $82.5 \mathrm{mM} \mathrm{NaCl}, 2.5 \mathrm{mM}$ $\mathrm{KCl}, 1.8 \mathrm{mM} \mathrm{CaCl}_{2}, 1 \mathrm{mM} \mathrm{MgCl}$ and $5 \mathrm{mM}$ HEPES buffered at $\mathrm{pH}$ 7.4. Cells were held at a fixed potential of $-80 \mathrm{mV}$ throughout the experiment. Agonist-evoked current responses were obtained by perfusing oocytes with a range of GABA concentrations in OR2 solution. Different nanobodies diluted into OR2 solution were tested at a range of concentrations by pre-incubation with nanobody alone and followed by a coapplication of nanobody with $5 \mathrm{mM}$ GABA. Data acquired with the HiClamp were analyzed using the manufacturer's software (Multi Channel Systems). Concentration403 activation curves were fitted with the empirical Hill equation as previously described 404 (Spurny et al. 2012). 
405

406

407

408

409

410

411

412

413

414

415

416

417

418

419

420

421

422

423

424

425

426

427

428

429

430

431

432

433

434

435

436

437

\section{ELIC purification and crystallization of ELIC-nanobody complexes}

Purified ELIC protein was produced as previously described, but with minor modifications (Spurny et al. 2012). In brief, the ELIC expression plasmid was transformed into the C43 E. coli strain and cells were grown in LB medium. Protein expression was induced with $200 \mu \mathrm{M}$ isopropyl $\beta$-D-1-thiogalactopyranoside (IPTG) and incubated in an orbital shaker at $20^{\circ} \mathrm{C}$ overnight. After cell lysis, membranes were collected by ultracentrifugation at $125,000 \times \mathrm{g}$ and solubilized with $2 \%(\mathrm{w} / \mathrm{v})$ anagrade n-undecyl- $\beta$-D-maltoside (UDM, Anatrace) at $4^{\circ} \mathrm{C}$ overnight. The cleared supernatant containing the solubilized MBP-ELIC fusion protein was purified by affinity chromatography on amylose resin (New England Biolabs). Column-bound ELIC was cleaved off by $3 \mathrm{CV}$ protease in the presence of $1 \mathrm{mM}$ EDTA $+1 \mathrm{mM}$ DTT at $4^{\circ} \mathrm{C}$ overnight. A final purification step was carried out on a Superdex 200 Increase 10/300 GL column (GE Healthcare) equilibrated with buffer containing $10 \mathrm{mM}$ Na-phosphate $\mathrm{pH}$ 8.0, $150 \mathrm{mM} \mathrm{NaCl}$, and 0.15\% n-undecyl- $\beta$-D-maltoside (UDM, Anatrace). Peak fractions containing pentameric ELIC were pooled, concentrated to $\sim 10 \mathrm{mg} / \mathrm{mL}$ and relipidated with $0.5 \mathrm{mg} / \mathrm{mL}$ E. coli lipids (Avanti Polar Lipids). Nanobodies were added at a $20 \%$ molar excess calculated for monomers and incubated at room temperature $2 \mathrm{~h}$ prior to setting up crystallization screens with a Mosquito liquid handling robot (TTP Labtech). Crystals for the ELIC complex with PAM-Nb grew at room temperature in the presence of $0.1 \mathrm{M} \mathrm{GABA}, 0.2 \mathrm{M} \mathrm{Ca}(\mathrm{OAc})_{2}, 0.1 \mathrm{M} \mathrm{MES}$ buffer $\mathrm{pH} 6.5$ and $10 \%$ PEG8000. Crystals for the ELIC complex with NAM-Nb grew at room temperature in the presence of $0.1 \mathrm{M} \mathrm{Na}_{2} \mathrm{SO}_{4}, 0.1 \mathrm{M}$ bis-trispropane $\mathrm{pH} 8.5$ and $10 \%$ PEG3350. Crystals were harvested after adding cryo-protectant containing mother liquor gradually supplemented with up to $25 \%$ glycerol in $5 \%$ increments. Crystals were then plunged into liquid nitrogen and stored in a dewar for transport to the synchrotron.

\section{Structure determination of ELIC-nanobody complexes}

Diffraction data for the ELIC+PAM-Nb structure were collected at the PROXIMA 1 beamline of the SOLEIL synchrotron (Gif-sur-Yvette, France). Diffraction data for the ELIC+NAM-Nb structure were collected at the X06A beamline of the Swiss Light Source (Villigen, Switzerland). Both structures were solved by molecular replacement with Phaser in the CCP4 suite (Winn et al. 2011) using the published structures for the ELIC pentamer (pdb 2vl0) and a GPCR nanobody (pdb 3p0g) as search templates. 
438 For the ELIC+PAM-Nb structure, the asymmetric unit contains one ELIC pentamer 439 with 5 nanobodies bound (one to each subunit). For the ELIC+NAM-Nb structure, the 440 asymmetric unit contains 2 ELIC pentamers with 1 nanobody bound to each pentamer.

441 The electron density for one of these nanobody molecules is not well defined, 442 suggesting partial occupancy at this pentamer, and therefore the atom occupancies 443 for this nanobody were manually set to $40 \%$ during structure refinement. The data set 444 for the ELIC+NAM-Nb was anisotropic with data extending to $\sim 3.15 \AA$ in the best direction and $\sim 3.5 \AA$ in the worst. To correct for anisotropy the unmerged reflections from XDS where uploaded to the STARANISO server (Paciorek et al. 2018) and automatically processed using $\mathrm{CC} 1 / 2>30$ and $\mathrm{I} / \sigma>2$ as resolution cut-off criteria. The merged and scaled data set from this procedure extends to a resolution of $3.25 \AA$ and the statistics produced by the STARANSIO server are shown in the crystallographic table (supplementary Table 1). Structures were improved by iterative rounds of manual rebuilding in Coot (Emsley et al. 2010) and automated refinement in Buster (Smart et al. 2012) or Refmac (Winn et al. 2011). Structure validation was carried out in PDB-REDO (Joosten et al. 2014) and MolProbity (V. B. Chen et al. 2010). Figures were prepared with PyMOL (Schrödinger). Pore radius profiles were made using CHAP (Klesse, n.d.). Simulated annealing omit maps were calculated in PHENIX and are shown for the nanobody-ELIC interaction region (supplementary Figure S1-S2).

458

\section{Cysteine-scanning mutagenesis and voltage-clamp recordings of $5-\mathrm{HT}_{3 \mathrm{~A}} \mathrm{R}$}

Stage V-VI Xenopus oocytes were purchased from Ecocyte (Germany) and stored in ND-96 (96 mM NaCl, 2 mM KCl, $1.8 \mathrm{mM} \mathrm{CaCl}_{2}, 1 \mathrm{mM} \mathrm{MgCl}_{2}, 5 \mathrm{mM} \mathrm{HEPES}$, $\mathrm{pH}$ 7.5) containing $2.5 \mathrm{mM}$ sodium pyruvate, $50 \mathrm{mM}$ gentamicin and $0.7 \mathrm{mM}$ 463 theophylline. cDNA encoding human 5- $\mathrm{HT}_{3 A} \mathrm{R}$ was cloned into the pGEM-HE expression plasmid (Liman, Tytgat, and Hess 1992). Mutants were engineered using the QuikChange mutagenesis kit (Agilent) and confirmed by sequencing. cRNA was in vitro transcribed from linearized pGEM-HE cDNA template using the mMessage mMachine T7 Transcription kit (ThermoFisher). Oocytes were injected with $50 \mathrm{nl}$ of 400 ng/ $\mu \mathrm{l}$ cRNA, and currents were recorded 18-48 hours post-injection. 5-HT $\mathrm{H}_{3 \mathrm{~A}} \mathrm{R}$ 
471 ND-96 with no added calcium, and 5-HT (creatinine sulphate complex, Sigma) was

472 diluted in this media for obtaining concentration-response curves. MTSEA-biotin

473 (Biotium) was diluted immediately prior to application into calcium-free ND-96 solution

474 at a concentration of $2 \mathrm{mM}$ from a stock solution of $500 \mathrm{mM}$ in DMSO. Analysis and

475 curve fitting was performed using Prism v4.03 (GraphPad Software). Concentration-

476 response data for each oocyte were normalized to the maximum current for that 477 oocyte.

\section{ACKNOWLEDGMENTS}

We thank beamline staff at the SOLEIL synchrotron and Swiss Light Source for assistance with data collection. SBO/IWT-project 1200261 and FWO-project G.0762.13 were awarded to JS and CU. Additional support was from KU Leuven OT/13/095, C32/16/035 and C14/17/093 to CU.

TRANSPARENT REPORTING STATEMENTS

All Xenopus electrophysiology experiments were repeated 4-8 times. The number of " $n$ " is mentioned in the relevant sections of the main text. We define each separate oocyte recording as a biological repeat. No data were excluded, unless the oocyte gave no detectable current. All electrophysiology experiments were conducted on automated devices, either the HiClamp or the Roboocyte, so essentially there was no human bias in recording of these data. Data are presented as the mean \pm standard error of the mean (SEM) with the raw data points overlaid as a dot plot. Statistical comparison between groups of data was performed using an unpaired two-tailed $t$ test and the significance value $P$ is mentioned in the relevant sections of the manuscript.

The X-ray diffraction data sets were collected from single crystals and typically the data set with the highest resolution was used for structural elucidation. Equivalent reflection data were recorded multiple times in agreement with the rotational symmetry of the crystal packing. The relevant data multiplicity value for each data set is mentioned in the crystallographic table (supplementary info). All aspects of X-ray data 
DATA AVAILABILITY

Atomic coordinates and structure factors have been deposited with the Protein

Data Bank under accession numbers 6SSI for the ELIC+PAM-Nb structure and 6SSP for the ELIC+NAM-Nb structure. The raw X-ray diffraction images for both data sets

deposited on

datadryad.org

under

accession number doi:10.5061/dryad.pv4097s. A source file is submitted with the data plotted in Figure 1a-b, Figure 2c and Figure 4c-d-e.

512

\section{FIGURE LEGENDS}

514 Figure 1. Nanobodies active as allosteric modulators and structures bound to the ELIC channel

516 a, b. Electrophysiological recordings of ELIC activated by the agonist GABA and in 517 the presence of increasing concentrations of PAM-Nb (a, green) or NAM-Nb (b, red).

518 The curve represents a fit to the Hill equation to the normalized current responses.

519 Circles represent averaged data with standard errors. c,d. X-ray crystal structures of 520 ELIC bound by PAM-Nb (c) or NAM-Nb (d). The cartoon representation shows a top521 down view onto the ELIC pentamer along the 5-fold axis (blue). e,f. Side views from 522 c,d. The dashed lines indicate the presumed location of the membrane boundaries.

Figure 2. Detailed nanobody interaction sites in ELIC and channel pore analysis

a. A detailed view of the interaction between PAM-Nb (green) and a single ELIC subunit (blue). The binding site for PAM-Nb overlaps with a known allosteric binding site for a small molecule fragment called CU2017 (ref. (Delbart et al. 2018), pdb code 5oui), bound between the $\beta 8-\beta 9$ strands (in orange) and the fragment shown as spheres (carbon yellow, nitrogen blue).

b. Detailed view of the interaction between NAM-Nb (red) and a single ELIC subunit (blue). The binding site for NAM-Nb involves a region which is part of a known allosteric binding site for flurazepam (ref. (Spurny et 
537 Figure 3. Distinct conformations of the vestibule site in pentameric ligand-gated 538 ion channels

539 a-c. Yellow ribbon representation of a single subunit ligand binding domain. Part of

540 the vestibule site (shown in blue cartoon), called the $\Omega$-loop, adopts 3 distinct conformations in different pLGICs: the $\Omega$-in (red, a), $\Omega$-out (green, b) and $\Omega$-open conformation (blue, c). d-f. Insets show a zoom of the $\Omega$-loop in surface representation to illustrate occluded vestibule site access in the $\Omega$-in and $\Omega$-out conformations, compared to an accessible vestibule site in the $\Omega$-open conformation. $\mathbf{g}$-i. Sequence alignment of the $\Omega$-loop and neighboring residues in pLGICs for which structures have been elucidated.

Figure 4. Allosteric modulation of the $5-\mathrm{HT}_{3} \mathrm{~A}$ receptor through chemical modification of engineered cysteines in the vestibule site

a. Example traces of agonist-evoked channel responses of the L151C 5-HT $3 A R$ mutant before and after modification with MTSEA-biotin show potentiation after cysteine modification. b. Location of L151C and other engineered cysteine mutants in this study, shown in ball\&stick representation. c. Concentration-activation curves before and after modification with MTSEA-biotin are a Hill curve fit for the recordings shown in (a) as well as additional data for T112C (d) and K149C. (c-e) Each of these 3 increase of the maximal current response.

\section{REFERENCES}

Althoff, Thorsten, Ryan E Hibbs, Surajit Banerjee, and Eric Gouaux. 2014. "X-Ray

Structures of GluCl in Apo States Reveal a Gating Mechanism of Cys-Loop Receptors.." Nature 512 (7514): 333-37. doi:10.1038/nature13669.

Basak, Sandip, Yvonne Gicheru, Shanlin Rao, Mark S P Sansom, and Sudha Chakrapani. 2018. "Cryo-EM Reveals Two Distinct Serotonin-Bound Conformations of Full-Length 5-HT3A Receptor.." Nature 563 (7730): 270-74. doi:10.1038/s41586-018-0660-7.

Bertrand, Daniel, and Murali Gopalakrishnan. 2007. "Allosteric Modulation of Nicotinic Acetylcholine Receptors." Biochemical Pharmacology 74 (8): 1155-63. doi:10.1016/j.bcp.2007.07.011.

Bocquet, Nicolas, Hugues Nury, Marc Baaden, Chantal Le Poupon, Jean-Pierre Changeux, Marc Delarue, and Pierre-Jean Corringer. 2009. "X-Ray Structure of a Pentameric Ligand-Gated Ion Channel in an Apparently Open Conformation." Nature 457 (7225): 111-14. doi:10.1038/nature07462. 
Brams, Marijke, Anshul Pandya, Dmitry Kuzmin, René Van Elk, Liz Krijnen, Jerrel L Yakel, Victor Tsetlin, August B Smit, and Chris Ulens. 2011. "A Structural and Mutagenic Blueprint for Molecular Recognition of Strychnine and D-Tubocurarine by Different Cys-Loop Receptors.." PLoS Biology 9 (3). Public Library of Science: e1001034. doi:10.1371/journal.pbio.1001034.

Brejc, K, W J van Dijk, R V Klaassen, M Schuurmans, J van Der Oost, A B Smit, and T K Sixma. 2001. "Crystal Structure of an ACh-Binding Protein Reveals the Ligand-Binding Domain of Nicotinic Receptors.." Nature 411 (6835): 269-76. doi:10.1038/35077011.

Celie, Patrick H N, Igor E Kasheverov, Dmitry Y Mordvintsev, Ronald C Hogg, Pim van Nierop, René Van Elk, Sarah E van Rossum-Fikkert, et al. 2005. "Crystal Structure of Nicotinic Acetylcholine Receptor Homolog AChBP in Complex with an Alpha-Conotoxin PnIA Variant.." Nature Structural \& Molecular Biology 12 (7): 582-88. doi:10.1038/nsmb951.

Chen, Qiang, Marta M Wells, Palaniappa Arjunan, Tommy S Tillman, Aina E Cohen, Yan Xu, and Pei Tang. 2018. "Structural Basis of Neurosteroid Anesthetic Action on GABAA Receptors.." Nature Communications 9 (1). Nature Publishing Group: 3972. doi:10.1038/s41467-018-06361-4.

Chen, Vincent B, W Bryan Arendall, Jeffrey J Headd, Daniel A Keedy, Robert M Immormino, Gary J Kapral, Laura W Murray, Jane S Richardson, and David C Richardson. 2010. "MolProbity: All-Atom Structure Validation for Macromolecular Crystallography..." Acta Crystallographica Section D, Biological Crystallography 66 (Pt 1): 12-21. doi:10.1107/S0907444909042073.

Delbart, Florian, Marijke Brams, Fabian Gruss, Sam Noppen, Steve Peigneur, Sandro Boland, Patrick Chaltin, et al. 2018. "An Allosteric Binding Site of the A7 Nicotinic Acetylcholine Receptor Revealed in a Humanized Acetylcholine-Binding Protein.." Journal of Biological Chemistry 293 (7). American Society for Biochemistry and Molecular Biology: 2534-45. doi:10.1074/jbc.M117.815316. Dellisanti, Cosma D, Yun Yao, James C Stroud, Zuo-Zhong Wang, and Lin Chen. 2007. "Crystal Structure of the Extracellular Domain of nAChR Alpha1 Bound to Alpha-Bungarotoxin at 1.94 a Resolution.." Nature Neuroscience 10 (8): 953-62. doi:10.1038/nn1942.

Du, Juan, Wei Lü, Shenping Wu, Yifan Cheng, and Eric Gouaux. 2015. "Glycine Receptor Mechanism Elucidated by Electron Cryo-Microscopy.." Nature 526 (7572): 224-29. doi:10.1038/nature14853.

Emsley, P, B Lohkamp, W G Scott, and K Cowtan. 2010. "Features and Development of Coot.." Acta Crystallographica Section D, Biological Crystallography 66 (Pt 4): 486-501. doi:10.1107/S0907444910007493.

Gill-Thind, JasKiran K, Persis Dhankher, Jarryl M D'Oyley, Tom D Sheppard, and Neil S Millar. 2015. "Structurally Similar Allosteric Modulators of A7 Nicotinic Acetylcholine Receptors Exhibit Five Distinct Pharmacological Effects.." Journal of Biological Chemistry 290 (6). American Society for Biochemistry and Molecular Biology: 3552-62. doi:10.1074/jbc.M114.619221.

Hassaine, Ghérici, Cédric Deluz, Luigino Grasso, Romain Wyss, Menno B Tol, Ruud Hovius, Alexandra Graff, et al. 2014. "X-Ray Structure of the Mouse Serotonin 5HT3 Receptor..” Nature 512 (7514): 276-81. doi:10.1038/nature13552.

Hibbs, Ryan E, and Eric Gouaux. 2011. "Principles of Activation and Permeation in an Anion-Selective Cys-Loop Receptor.." Nature 474 (7349): 54-60. doi:10.1038/nature10139. 
624

625

626

627

628

629

630

631

632

633

634

635

636

637

638

639

640

641

642

643

644

645

646

647

648

649

650

651

652

653

654

655

656

657

658

659

660

661

662

663

664

665

666

667

668

669

670

671

672
Hilf, Ricarda J C, and Raimund Dutzler. 2008. "X-Ray Structure of a Prokaryotic Pentameric Ligand-Gated Ion Channel.." Nature 452 (7185): 375-79. doi:10.1038/nature06717.

Hilf, Ricarda J C, and Raimund Dutzler. 2009. "Structure of a Potentially Open State of a Proton-Activated Pentameric Ligand-Gated Ion Channel." Nature 457 (7225): 115-18. doi:10.1038/nature07461.

$\mathrm{Hu}$, Haidai, Akos Nemecz, Catherine Van Renterghem, Zaineb Fourati, Ludovic Sauguet, Pierre-Jean Corringer, and Marc Delarue. 2018. "Crystal Structures of a Pentameric Ion Channel Gated by Alkaline pH Show a Widely Open Pore and Identify a Cavity for Modulation.." Proceedings of the National Academy of Sciences, April. National Academy of Sciences, 201717700. doi:10.1073/pnas.1717700115.

Huang, Xin, Hao Chen, Klaus Michelsen, Stephen Schneider, and Paul L Shaffer. 2015. "Crystal Structure of Human Glycine Receptor-A3 Bound to Antagonist Strychnine.." Nature 526 (7572): 277-80. doi:10.1038/nature14972.

Joosten, Robbie P, Fei Long, Garib N Murshudov, and Anastassis Perrakis. 2014. "The PDB_REDO Server for Macromolecular Structure Model Optimization.." IUCrJ 1 (Pt 4): 213-20. doi:10.1107/S2052252514009324.

Karlin, A, and M H Akabas. 1998. "Substituted-Cysteine Accessibility Method.." Methods in Enzymology 293: 123-45. doi:10.1016/S0076-6879(98)93011-7.

Klesse, Gianni. n.d. "CHAP: a Versatile Tool for the Structural and Functional Annotation of Ion Channel Pores." bioRxiv 35: 527275. doi:10.1101/527275.

Knoflach, Frédéric, Maria-Clemencia Hernandez, and Daniel Bertrand. 2018. "Methods for the Discovery of Novel Compounds Modulating a GammaAminobutyric Acid Receptor Type a Neurotransmission.." Journal of Visualized Experiments : JoVE, no. 138 (August): e57842. doi:10.3791/57842.

Kouvatsos, Nikolaos, Petros Giastas, Dafni Chroni-Tzartou, Cornelia Poulopoulou, and Socrates J Tzartos. 2016. "Crystal Structure of a Human Neuronal nAChR Extracellular Domain in Pentameric Assembly: Ligand-Bound A2 Homopentamer.." Proceedings of the National Academy of Sciences 113 (34). National Academy of Sciences: 9635-40. doi:10.1073/pnas.1602619113.

Laverty, Duncan, Philip Thomas, Martin Field, Ole J Andersen, Matthew G Gold, Philip C Biggin, Marc Gielen, and Trevor G Smart. 2017. "Crystal Structures of a GABAA-Receptor Chimera Reveal New Endogenous Neurosteroid-Binding Sites.." Nature Structural \& Molecular Biology 24 (11): 977-85. doi:10.1038/nsmb.3477.

Laverty, Duncan, Rooma Desai, Tomasz Uchański, Simonas Masiulis, Wojciech J Stec, Tomas Malinauskas, Jasenko Zivanov, et al. 2019. "Cryo-EM Structure of the Human A1ß3y2 GABAA Receptor in a Lipid Bilayer.." Nature 565 (7740): 516-20. doi:10.1038/s41586-018-0833-4.

Li, Shu-Xing, Sun Huang, Nina Bren, Kaori Noridomi, Cosma D Dellisanti, Steven M Sine, and Lin Chen. 2011. "Ligand-Binding Domain of an A7-Nicotinic Receptor Chimera and Its Complex with Agonist.." Nature Neuroscience 14 (10). Nature Publishing Group: 1253-59. doi:10.1038/nn.2908.

Liman, E R, J Tytgat, and P Hess. 1992. "Subunit Stoichiometry of a Mammalian K+ Channel Determined by Construction of Multimeric cDNAs." Neuron 9 (5): $861-$ 71.

Lummis, Sarah C R. 2012. "5-HT3 Receptors.." Journal of Biological Chemistry, October. doi:10.1074/jbc.R112.406496. 
Manglik, Aashish, Brian K Kobilka, and Jan Steyaert. 2017. "Nanobodies to Study G Protein-Coupled Receptor Structure and Function.." Annual Review of Pharmacology and Toxicology 57 (January): 19-37. doi:10.1146/annurevpharmtox-010716-104710.

Masiulis, Simonas, Rooma Desai, Tomasz Uchański, Itziar Serna Martin, Duncan Laverty, Dimple Karia, Tomas Malinauskas, et al. 2019. "GABAA Receptor Signalling Mechanisms Revealed by Structural Pharmacology.." Nature 565 (7740): 454-59. doi:10.1038/s41586-018-0832-5.

Miller, Paul S, and A Radu Aricescu. 2014. "Crystal Structure of a Human GABAA Receptor.." Nature 512 (7514): 270-75. doi:10.1038/nature13293.

Miller, Paul S, Suzanne Scott, Simonas Masiulis, Luigi De Colibus, Els Pardon, Jan Steyaert, and A Radu Aricescu. 2017. "Structural Basis for GABAA Receptor Potentiation by Neurosteroids.." Nature Structural \& Molecular Biology 24 (11). Nature Publishing Group: 986-92. doi:10.1038/nsmb.3484.

Miyazawa, Atsuo, Yoshinori Fujiyoshi, and Nigel Unwin. 2003. "Structure and Gating Mechanism of the Acetylcholine Receptor Pore.." Nature 423 (6943): 949-55. doi:10.1038/nature01748.

Monod, J, J Wyman, and J P Changeux. 1965. "On the Nature of Allosteric Transitions: a Plausible Model.." Journal of Molecular Biology 12 (May): 88-118. Morales-Perez, Claudio L, Colleen M Noviello, and Ryan E Hibbs. 2016. "X-Ray Structure of the Human A4ß2 Nicotinic Receptor.." Nature 538 (7625). Nature Publishing Group: 411-15. doi:10.1038/nature19785.

Nury, Hugues, Catherine Van Renterghem, Yun Weng, Alphonso Tran, Marc Baaden, Virginie Dufresne, Jean-Pierre Changeux, James M Sonner, Marc Delarue, and Pierre-Jean Corringer. 2011. "X-Ray Structures of General Anaesthetics Bound to a Pentameric Ligand-Gated Ion Channel.." Nature 469 (7330): 428-31. doi:10.1038/nature09647.

Nys, Mieke, Eveline Wijckmans, Ana Farinha, Özge Yoluk, Magnus Andersson, Marijke Brams, Radovan Spurny, et al. 2016. "Allosteric Binding Site in a CysLoop Receptor Ligand-Binding Domain Unveiled in the Crystal Structure of ELIC in Complex with Chlorpromazine.." Proceedings of the National Academy of Sciences 113 (43). National Acad Sciences: E6696-E6703. doi:10.1073/pnas.1603101113.

Paciorek, W, A Sharff, C Vonrhein, and G Bricogne. 2018. STARANISO, Cambridge, United Kingdom: Global Phasing Ltd.

Pardon, Els, Toon Laeremans, Sarah Triest, Søren G F Rasmussen, Alexandre Wohlkönig, Armin Ruf, Serge Muyldermans, Wim G J Hol, Brian K Kobilka, and Jan Steyaert. 2014. "A General Protocol for the Generation of Nanobodies for Structural Biology.." Nature Protocols 9 (3). Nature Publishing Group: 674-93. doi:10.1038/nprot.2014.039.

Phulera, Swastik, Hongtao Zhu, Jie Yu, Derek P Claxton, Nate Yoder, Craig Yoshioka, and Eric Gouaux. 2018. "Cryo-EM Structure of the BenzodiazepineSensitive $\alpha 1 \beta 1 y 2 S$ Tri-Heteromeric GABAA Receptor in Complex with GABA.." eLife 7 (July): 531. doi:10.7554/eLife.39383.

Polovinkin, Lucie, Ghérici Hassaine, Jonathan Perot, Emmanuelle Neumann, Anders A Jensen, Solène N Lefebvre, Pierre-Jean Corringer, et al. 2018.

"Conformational Transitions of the Serotonin 5-HT3 Receptor.." Nature 563 (7730): 275-79. doi:10.1038/s41586-018-0672-3.

Sauguet, Ludovic, Rebecca J Howard, Laurie Malherbe, Ui S Lee, Pierre-Jean Corringer, R Adron Harris, and Marc Delarue. 2013. "Structural Basis for 
Potentiation by Alcohols and Anaesthetics in a Ligand-Gated Ion Channel.." Nature Communications 4 (April): 1697. doi:10.1038/ncomms2682.

Scully, Marie, Spero R Cataland, Flora Peyvandi, Paul Coppo, Paul Knöbl, Johanna A Kremer Hovinga, Ara Metjian, et al. 2019. "Caplacizumab Treatment for Acquired Thrombotic Thrombocytopenic Purpura.." The New England Journal of Medicine 380 (4): 335-46. doi:10.1056/NEJMoa1806311.

Smart, Oliver S, Thomas O Womack, Claus Flensburg, Peter Keller, Włodek Paciorek, Andrew Sharff, Clemens Vonrhein, and Gérard Bricogne. 2012. "Exploiting Structure Similarity in Refinement: Automated NCS and TargetStructure Restraints in BUSTER.." Acta Crystallographica Section D, Biological Crystallography 68 (Pt 4). International Union of Crystallography: 368-80. doi:10.1107/S0907444911056058.

Spurny, Radovan, Bert Billen, Rebecca J Howard, Marijke Brams, Sarah Debaveye, Kerry L Price, David A Weston, et al. 2013. "Multisite Binding of a General Anesthetic to the Prokaryotic Pentameric Erwinia Chrysanthemi Ligand-Gated Ion Channel (ELIC).." Journal of Biological Chemistry 288 (12): 8355-64. doi:10.1074/jbc.M112.424507.

Spurny, Radovan, Joachim Ramerstorfer, Kerry Price, Marijke Brams, Margot Ernst, Hugues Nury, Mark Verheij, et al. 2012. "Pentameric Ligand-Gated Ion Channel ELIC Is Activated by GABA and Modulated by Benzodiazepines.." Proceedings of the National Academy of Sciences 109 (44): E3028-34. doi:10.1073/pnas.1208208109.

Spurny, Radovan, Sarah Debaveye, Ana Farinha, Ken Veys, Ann M Vos, Thomas Gossas, John Atack, et al. 2015. "Molecular Blueprint of Allosteric Binding Sites in a Homologue of the Agonist-Binding Domain of the A7 Nicotinic Acetylcholine Receptor.." Proceedings of the National Academy of Sciences 112 (19): E254352. doi:10.1073/pnas.1418289112.

Unwin, Nigel. 2005. "Refined Structure of the Nicotinic Acetylcholine Receptor at 4A Resolution.." Journal of Molecular Biology 346 (4): 967-89. doi:10.1016/j.jmb.2004.12.031.

Walsh, Richard M, Soung-Hun Roh, Anant Gharpure, Claudio L Morales-Perez, Jinfeng Teng, and Ryan E Hibbs. 2018. "Structural Principles of Distinct Assemblies of the Human A4ß2 Nicotinic Receptor..” Nature 557 (7704): 261-65. doi:10.1038/s41586-018-0081-7.

Winn, Martyn D, Charles C Ballard, Kevin D Cowtan, Eleanor J Dodson, Paul Emsley, Phil R Evans, Ronan M Keegan, et al. 2011. "Overview of the CCP4 Suite and Current Developments." Acta Crystallographica Section D, Biological Crystallography 67 (4). International Union of Crystallography: 235-42. doi:10.1107/S0907444910045749.

Zhu, Shaotong, Colleen M Noviello, Jinfeng Teng, Richard M Walsh, Jeong Joo Kim, and Ryan E Hibbs. 2018. "Structure of a Human Synaptic GABAA Receptor.." Nature 559 (7712). Nature Publishing Group: 67-72. doi:10.1038/s41586-0180255-3.

Zouridakis, Marios, Petros Giastas, Eleftherios Zarkadas, Dafni Chroni-Tzartou, Piotr Bregestovski, and Socrates J Tzartos. 2014. "Crystal Structures of Free and Antagonist-Bound States of Human." Nature Structural \& Molecular Biology 21 (11). Nature Publishing Group: 976-80. doi:10.1038/nsmb.2900. 


\section{Supplementary information}

773

\begin{tabular}{|c|c|c|}
\hline & ELIC+PAM-Nb & $\mathrm{ELIC}+\mathrm{NAM}-\mathrm{Nb}$ \\
\hline \multicolumn{3}{|l|}{ Crystallographic statistics } \\
\hline Beamline & PROXIMA 1 (SOLEIL) & X06A (SLS) \\
\hline Wavelength $(\AA)$ & 0.97857 & 0.9999 \\
\hline Spacegroup & $P 2_{1}$ & $P 1$ \\
\hline$a, b, c(\AA)$ & $105.6,146.56,140.24$ & $121.73,122.29,128.15$ \\
\hline$\alpha, \beta, \gamma\left({ }^{\circ}\right)$ & $90,111.39,90$ & $71.02,64.20,61.66$ \\
\hline Resolution limits $(\AA)$ & $49.67-2.59(2.65-2.59)$ & $48.32-3.25(3.34-3.25)$ \\
\hline$R_{\text {merge }}(\%)$ & $6.1(122.1)$ & $5.2(95.4)$ \\
\hline$R_{\text {meas }}(\%)$ & $7.8(157.1)$ & $7.4(134.9)$ \\
\hline$R_{\text {pim }}(\%)$ & $4.9(97.8)$ & $5.2(95.3)$ \\
\hline$<\mid / \sigma>$ & $13.4(1.0)$ & $5.5(0.5)$ \\
\hline$C C_{1 / 2}(\%)$ & $99.9(45.8)$ & $99.7(42.9)$ \\
\hline Multiplicity & $4.8(4.6)$ & $1.8(1.6)$ \\
\hline Completeness (\%) & $99.7(95.2)$ & $95.1(89.4)$ \\
\hline Total number of reflections & $594559(26957)$ & $147646(6664)$ \\
\hline Number unique reflections & $123770(5799)$ & $83183(4160)$ \\
\hline \multicolumn{3}{|c|}{ Refinement and model statistics } \\
\hline $\mathrm{R}_{\text {work }}(\%)$ & 22.97 & 24.80 \\
\hline $\mathrm{R}_{\text {free }}(\%)$ & 24.59 & 26.43 \\
\hline Rmsd bond distance $(\AA)$ & 0.008 & 0.008 \\
\hline Rmsd bond angle $\left({ }^{\circ}\right)$ & 0.94 & 0.97 \\
\hline \multicolumn{3}{|l|}{ Ramachandran analysis } \\
\hline Outliers (\%) & 0.05 & 0.16 \\
\hline Favored (\%) & 97.16 & 96.53 \\
\hline Poor rotamers (\%) & 0.63 & 0.77 \\
\hline Molprobity score & 1.54 (100rd percentile) & 1.78 (100rd percentile) \\
\hline
\end{tabular}




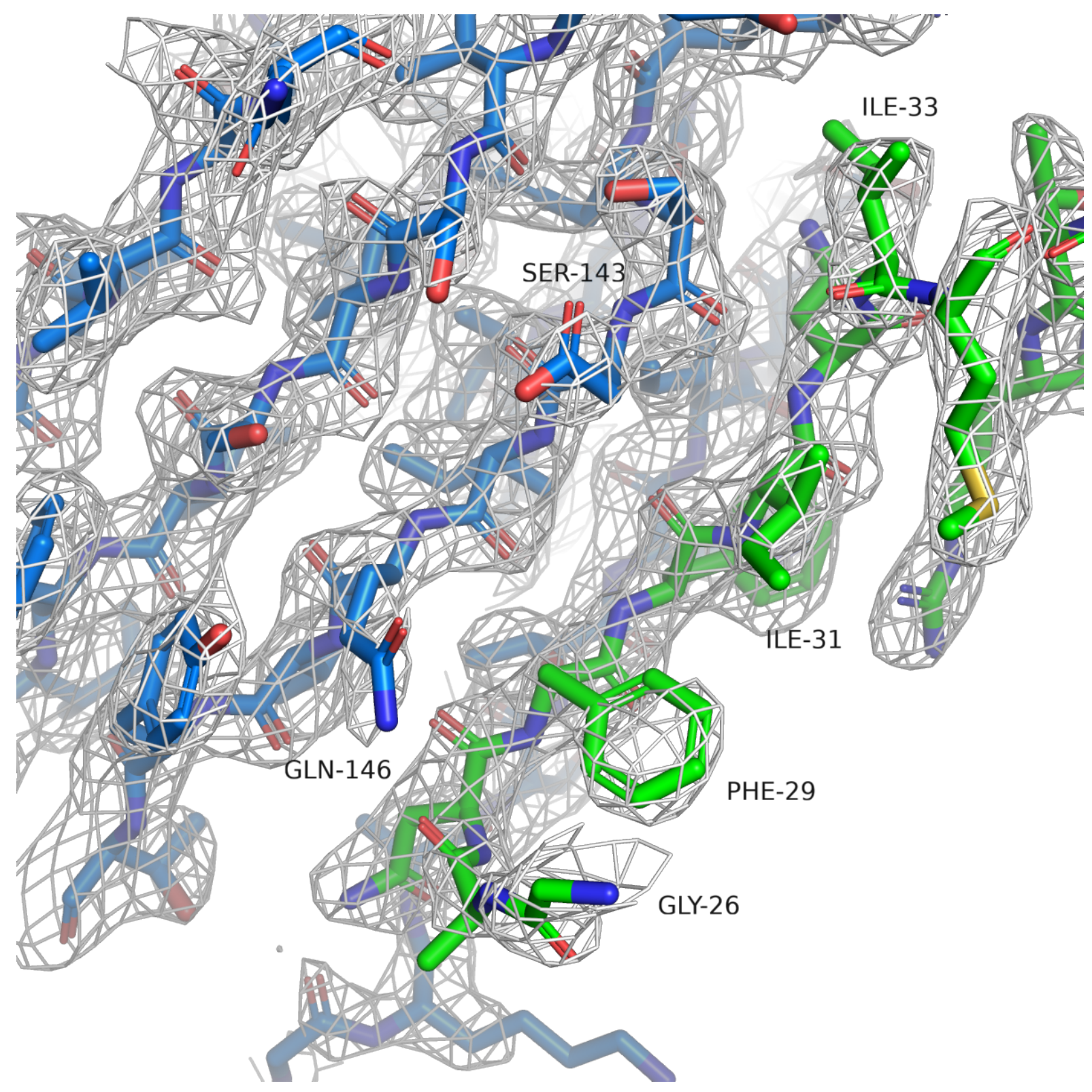

Supplementary Figure S1.

783 Simulated annealing omit map for ELIC+PAM-Nb structure. The white mesh shows a simulated annealing omit map calculated in PHENIX and contoured at a sigma level of 1.0 around a $12 \AA$ sphere zoom of the ELIC-Nb interface. In the omit calculation an omit box around the entire chain $\mathrm{F}$ (green) of the PAM-Nb was selected. ELIC is shown in blue and selected residues of the interaction interface are indicated. 


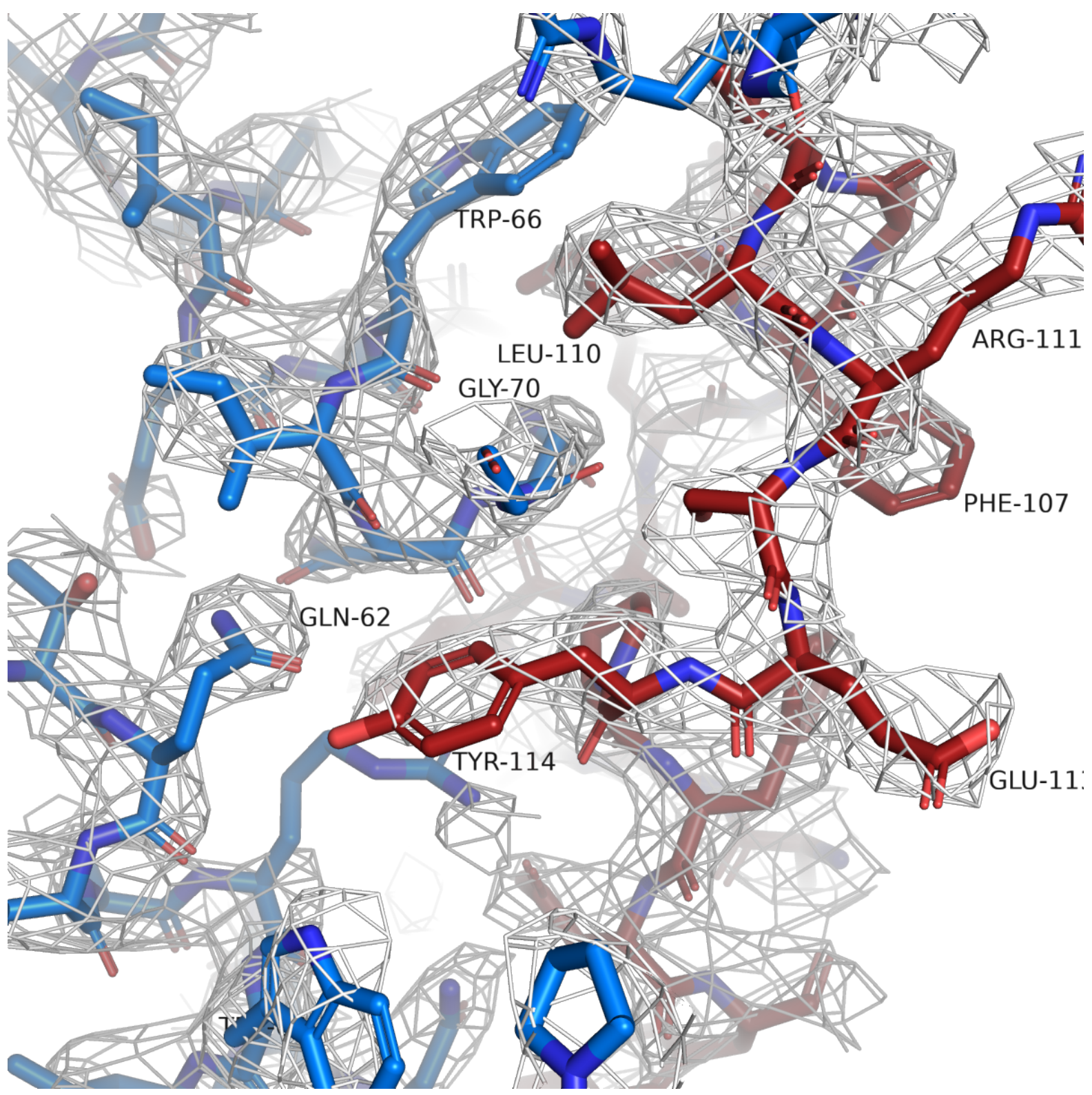

\section{Supplementary Figure S2.}

795 Simulated annealing omit map for ELIC+NAM-Nb structure. The white mesh shows a 796 simulated annealing omit map calculated in PHENIX and contoured at a sigma level 797 of 1.0 around a $12 \AA$ sphere zoom of the ELIC-Nb interface. In the omit calculation an 798 omit box around the entire chain $\mathrm{K}$ (red) of the PAM-Nb was selected. ELIC is shown in blue and selected residues of the interaction interface are indicated. 


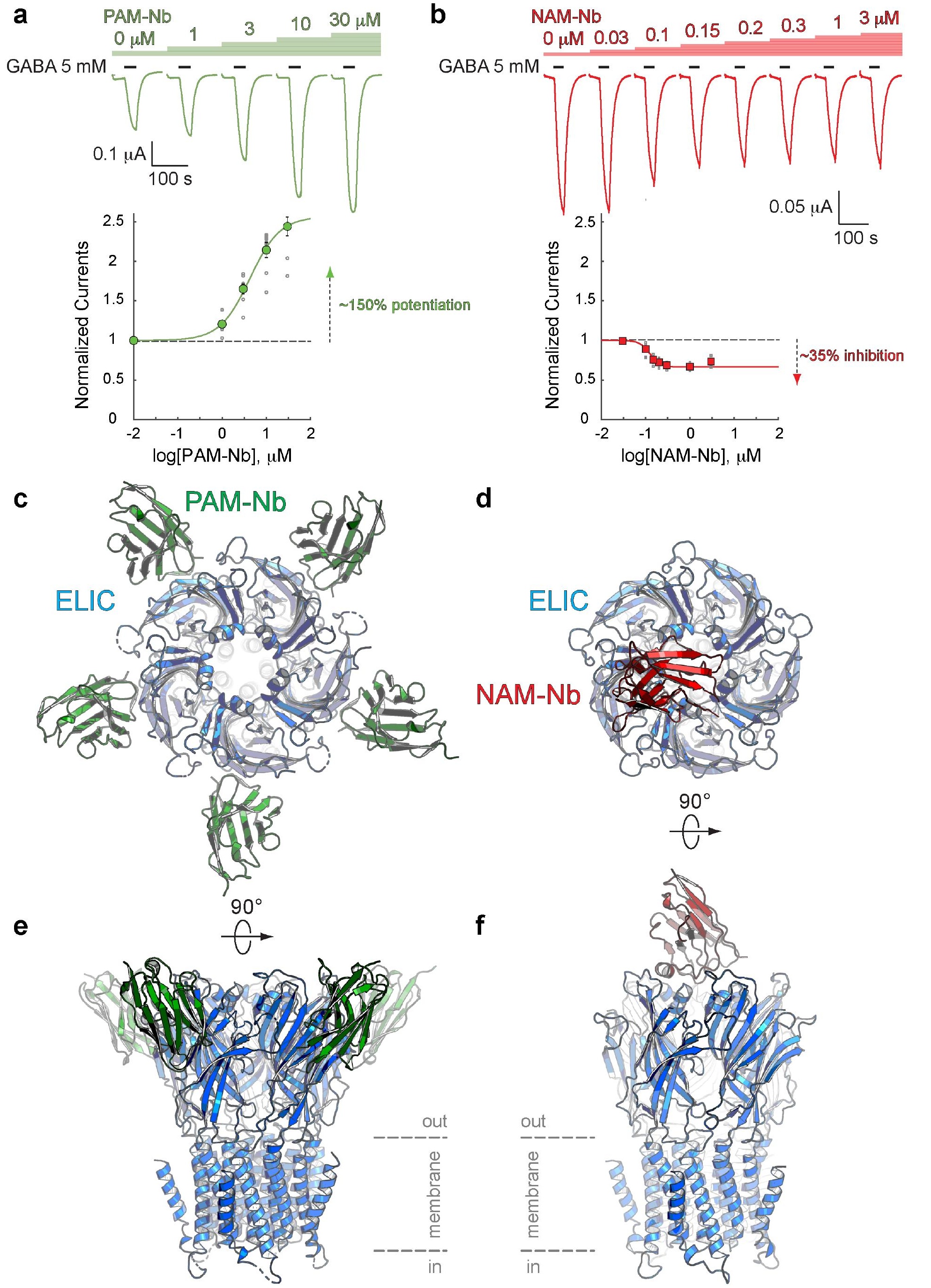



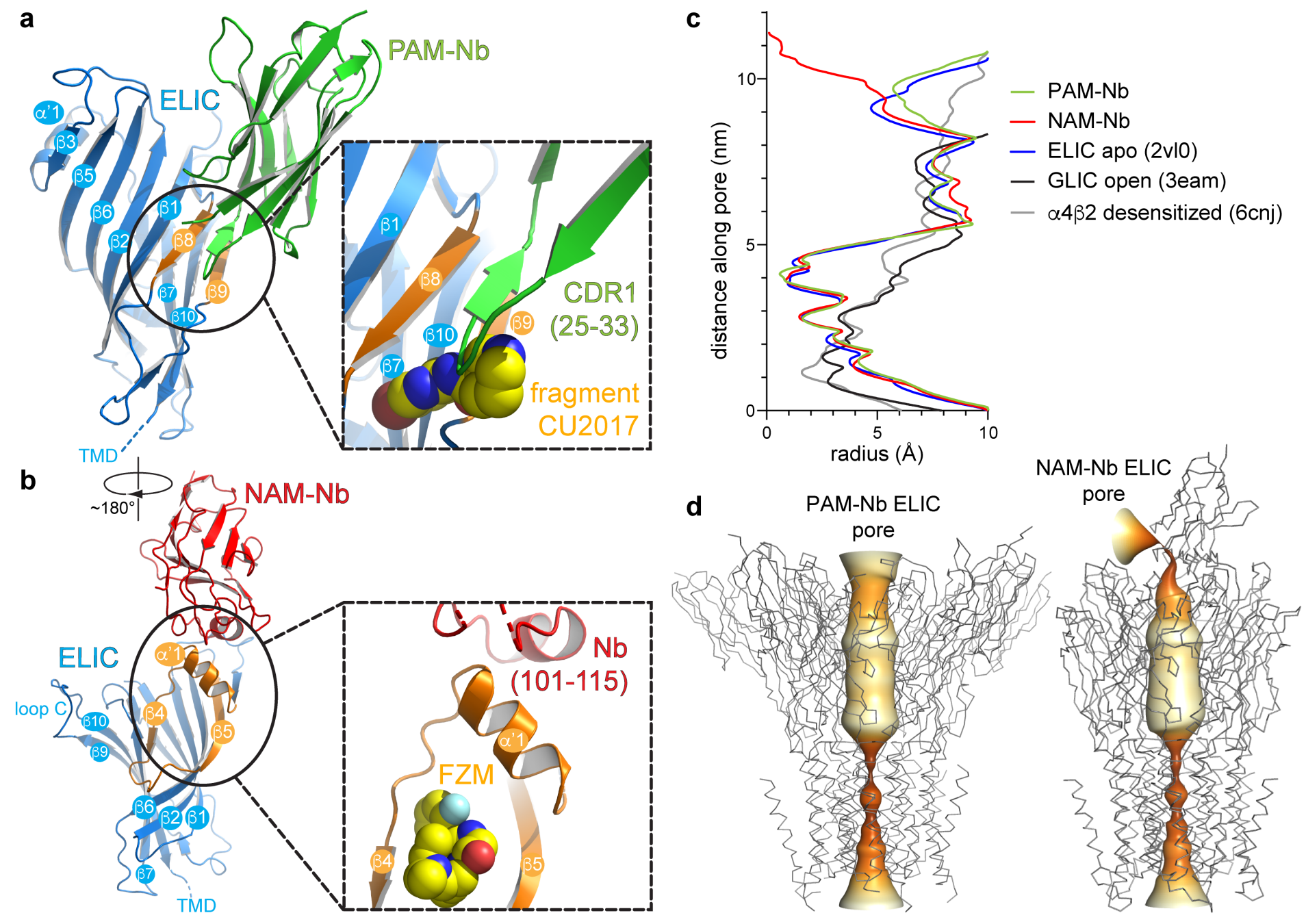
a

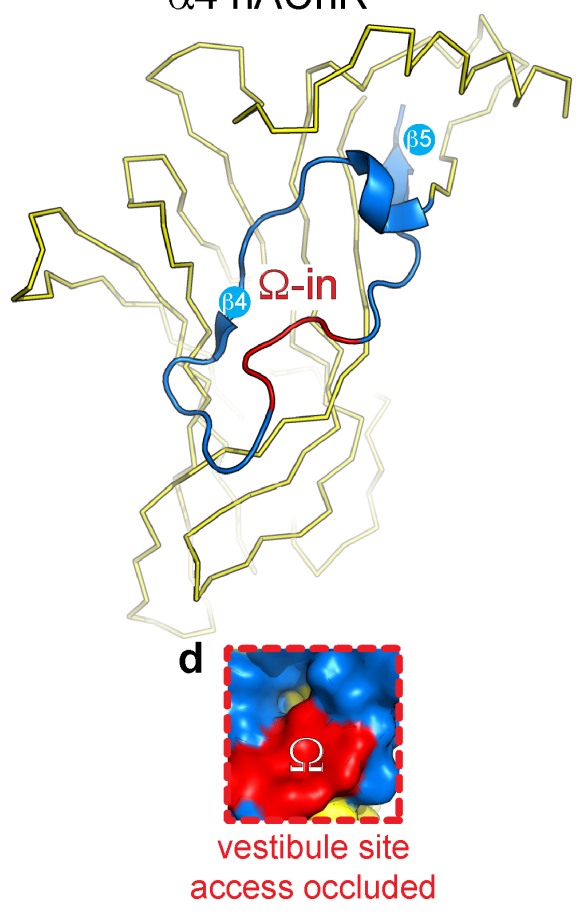

helix

ß4 $\Omega$-in $\beta 5$ nACh 2 PSEMI WI PDI VL YNNADGEFAVTHMT KAHLF nACh 4 PSEL I WRPDI VL YNNADGDFAVTHLTKAHLF nACh 32 PS KHLWLPDVVL YNNADGMYEVS F Y SNAVVS 5-HT3 PTDS IWVPDI L INEF VDVGKS - PN I P YVYVH b

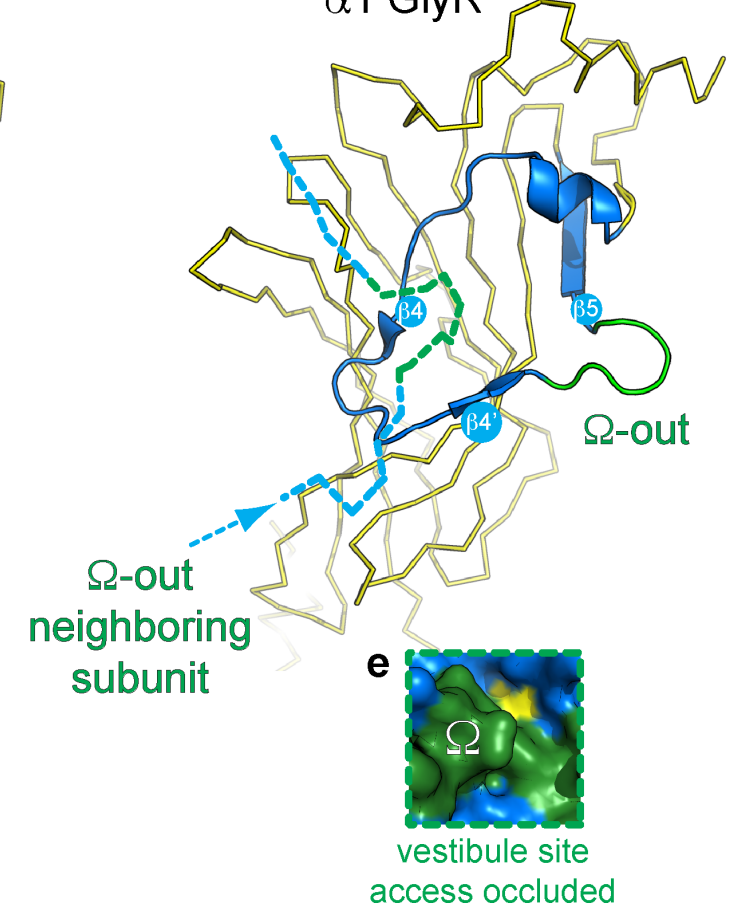

h

helix

$\beta 4 \quad \beta 4$ ' $\Omega$-out $\beta 5$

GABAB1 NRVADQLWVPDTYFLNDKKSF VHGVTVKNRMIRL GABAB2 NRVADQLWVPDTYFL NDKKSFVHGVTVKNRMIRL GABAB3 NRVADQLWVPDTYFL NDKKSF VHGVTVKNRMIRL GABAY2L S NMVG KI WIPDTFER SS KKADA HW I TTPNRMLRI GABA 1 NLMAS K I WTPDTF F HNGKK S VA HNMTM PNKL LR I Glyo1 P S MLDS I WKPDLFEANE KGANFHE VTTDNKLLR I Glyo3 P SMLDS I WKPDLFFANEKGANFHEVTTDNKLLR I GluCl - - VGHQI WMPDTFEPNE KQAYKHTIDKPNVL IR I 5-HT3 S I PTDS IWVPDI L INEF VDVGKS - - PN I PYYVYV
C

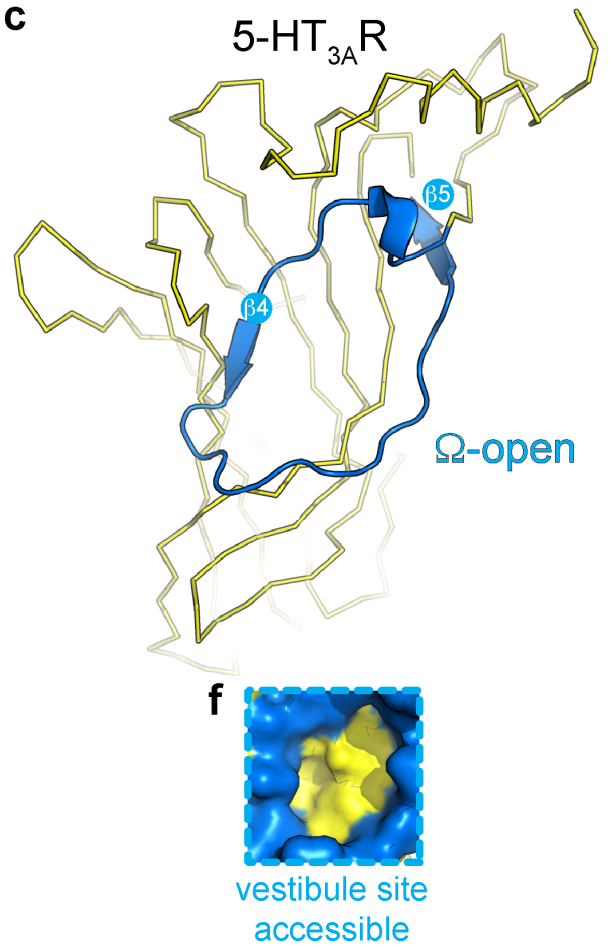

i

helix $\quad \beta 4 \quad \Omega$-open $\beta 5$

5-HT3A P TDS I WVPD I L I NEF VDVGKS - PN I PYVYV TMY PSEL LWLPDVVLENN VDCQF E VAYYANVLV TMS RPEL I WIPD I VL ONNNDGQYNVAYFCNVLV TM $\beta$ P S DDVWWQP I VLMNNNDCS FE I TLHVNVLV Tm $\alpha$ P S DD VWL $L$ P L VL YNNADGDFA I VHMTKL L L nACh 1 1 PS EK I WRPDVVL YNNADCDFA I VKF TKVLL nACha9 PS DL VWRPD I VL YNNKADDEE - P VNTNVVL

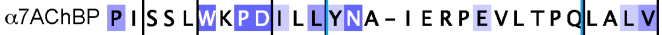
LSAChBP PISS LWVPDLAAYNA - I S KPEVLTPQLARV ELIC I NNG L WVPALLE I NV-VGSP DTGNK-RLML GLIC EPEA I WI PEIIRFVNV - ENARDADDVVDISV 
